\title{
LEXICOGRAFÍA ORTEGUIANA. MÁS SOBRE LA ELABORACIÓN DE CONCORDANCIAS LEMATIZADAS Y LA EDICIÓN DE TEXTOS
}

\author{
JAVIER FRESNILLO NÚÑEZ \\ Universidad de Alicante \\ javier.fresnillo@ua.es
}

\begin{abstract}
Resumen
En el presente artículo se hacen una serie de consideraciones generales sobre los criterios que, a nuestro juicio, son pertinentes a la hora de editar los textos de José Ortega y Gasset. En un aspecto concreto, el de las variantes textuales, hemos analizado tres ediciones distintas de El tema de nuestro tiempo utilizando herramientas informáticas (digitalización, OCR, etc.). La conclusión es que el texto orteguiano presenta variantes que no son recogidas en su totalidad por los editores.
\end{abstract}

PALABRAS CLAVE: edición, OCR, herramientas informáticas.

\begin{abstract}
In this article there can be found some general considerations on the criteria for the edition of José Ortega y Gasset's texts. Considering a special aspect of textual versions, three different editions of El tema de nuestro tiempo have been analyzed, using computer tools (digitalization, OCR, etc.). The conclusion is that the text by Ortega presents variants that are not entirely collected by the publishers.
\end{abstract}

KEYWORDS: edition, OCR, computer tools.

\section{Introducción}

Desde finales del año 2004 viene publicándose una nueva edición de las obras completas de José Ortega y Gasset ${ }^{1}$. Hasta el momento han salido a la luz siete volúmenes (el último en 2007); con el sexto quedó editada la obra publicada en vida del autor, mientras que a partir del volumen séptimo y hasta el décimo van a ir publicándose los trabajos que quedaron inéditos desde su fallecimiento.

En octubre de 2004 publicamos nosotros la Concordantia Ortegiana2. La concordancia está lematizada3. Para su realización tomamos como base la edición de Paulino Garagorri, culminada en 1983. Su título es, también, el de Obras completas.

\footnotetext{
${ }^{1}$ José Ortega y Gasset, Obras completas. Tomo i (1902-1915). Ed. Fog. (Carmen Asenjo Pinilla, Ignacio Blanco Alfonso, José Ramón Carriazo Ruiz, María Isabel Ferreiro Lavedán, Iñaki Gabaráin Gaztelumendi, Azucena López Cobo, Juan Padilla Moreno, Javier Zamora Bonilla), Taurus - Revista de Occidente, Madrid 2004. El Tomo ii (1916) tiene los mismos datos. Estos mismos investigadores han editado los tomos iii (1917-1925, publicado en 2005, dos ediciones), iv (1926-1931, publicado en 2005), v (1932-1940, publicado en 2006) y vi (1941-1955, publicado en 2006). En la edición del tomo vii (obra póstuma, 1902-1925, publicado en 2007) han intervenido Carmen Asenjo Pinilla, Cristina Blas Nistal, José Ramón Carriazo Ruiz, María Isabel Ferreiro Lavedán, Iñaki Gabaráin Gaztelumendi, Felipe González Alcázar, Azucena López Cobo, Javier Zamora Bonilla.

2 Javier Fresnillo Núñez - Fernando M. Pérez Herranz (col.). Concordantia Ortegiana. Concordantia in José Ortega y Gasset opera omnia. Universidad, Alicante 2004. El índice y la presentación están en la página del
} 


\section{Consideraciones generales}

A pesar de que nosotros, en nuestra calidad de lexicógrafos, no somos propiamente editores, sí que nos vemos obligados en ocasiones a subsanar erratas de la edición sobre la que hemos realizado nuestro trabajo. No obstante, esta tarea, aunque tenga elementos en común con la del editor, no es comparable a la de éste, por cuanto la persona que realiza la concordancia no está llevando a cabo una nueva edición, sino que tan sólo intenta subsanar un texto que -por una u otra razón- considera erróneo. Y ello no es nunca en detrimento del mérito del editor: simplemente, se trata de contribuir a la mejora de un trabajo previo.

En algunos casos el error detectado en la edición no afecta a la lematización. En este caso, el investigador consigna la errata en aras de la verdad científica. En la cita que encabeza el vol. II de El espectador (1917) Garagorri edita lo siguiente: pro)j to\n bi/on... kaqa/per toco/tai skopo\n e) \xontej "Seamos con nuestras vidas como arqueros que tienen un blanco". En pro/j debe ir un acento grave, no un espíritu suave; en e)/xontej debe ir un acento agudo, no uno grave; en la traducción, tras "vidas" deberían aparecer los mismos puntos suspensivos que en el texto griego ${ }^{4}$. Ninguna de las erratas señaladas afectan a la lematización.

En otros casos, por el contrario, la errata sí afecta a la lematización, y en este caso el investigador se ve impelido a corregirla, so pena de inventar un lema inexistente. En el texto precedente, que en Fog figura en la página 263 del tomo II, los editores de las nuevas obras completas cometen un error, al convertir toco/tai (“arqueros”) en *tozo/tai ${ }^{5}$. Pudiera parecer una errata, pero el error se distingue de la eventual errata en que aquél se repite ${ }^{6}$ mientras que ésta es ocasional. Y, en efecto, en la página 355 de su edición (tomo II) Fog edita to/zon ${ }^{7}$ y tozo/tai, en vez de las formas correctas to/cou y toco/tai, respectivamente ${ }^{8}$. En conclusión, si la errata altera el caso gramatical en que aparece la palabra, ello no incide en la lematización (el paradigma es el mismo). En cambio, si de la errata resulta un barbarismo (como es el caso de *to/zon, *tozo/tai), el lexicógrafo deberá lematizar la forma correcta, so pena de incurrir en el mismo error que los editores ${ }^{9}$.

servicio de Publicaciones de la Universidad de Alicante:

http://publicaciones.ua.es/publica/ficha.aspx?fndCod=LI9788479088019 [consulta: 04/02/2008].

${ }^{3}$ Op. cit. en la nota 2, págs. 19-24 (4. La lematización).

${ }^{4}$ Nosotros, en la Concordantia Ortegiana, omitimos -erróneamente- la cita por considerarla nota del editor. El texto figura en la página 127 del tomo ii de Garagorri. Ese mismo texto aparece nuevamente al comienzo del tomo iii de El espectador, en la página 226 del mismo tomo, y Garagorri lo edita sin errata ninguna.

${ }^{5}$ Marcamos con asterisco las formas no documentadas.

${ }^{6}$ Si leemos "néoterique" una vez, puede considerarse errata; si un autor escribe sistemáticamente "néoterique" en todas las ocasiones, podemos pensar que se trata de un error.

${ }^{7}$ En vez de to/cou, la forma correcta que edita Garagorri. El error es doble: $\mathrm{z}$ en vez de c, n en vez de u (la forma ha de ir en genitivo, en el mismo caso que lu/rhj.

${ }^{8}$ En Garagorri, como ya he dicho, en este caso se edita el texto sin erratas en ambos términos. El griego del texto orteguiano, en la edición de Fog., está especialmente deturpado en el tomo vii, que contiene textos orteguianos no incluidos en la edición de Garagorri.

${ }^{9}$ Curiosamente, en la pág. 135 de la traducción al castellano de Lo trágico. El lenguaje de Karl Jaspers (Ágora, Málaga 1995 [1948], preliminar y traducción de José Luis del Barco) leemos cwon lo/gon e)/xon. Se ha producido la misma confusión pero en sentido inverso, cwon en vez de la forma correcta $\mathrm{zw}=\mid \mathrm{on}$. Como dato curioso, diremos que una búsqueda en Google nos ha devuelto zoion, zôion, zoon y zôon. Un editor no debe permitirse estas 
No obstante, es preciso proceder con sumo respeto ante el texto editado, por razones de elemental prudencia. En ocasiones, el investigador se ve movido a modificar un texto aparentemente "incorrecto", pero hemos de actuar con cautela. En uno de los textos citados, el de Heráclito ${ }^{10}$, vemos formas dialectales griegas: o(/kwj (jónico-ático o(/pwj), e(wutw=| (jónico-ático e(autw=|), o(/kwsper (jónico-ático o(/pwsper). Hay que refrenar, pues, el impulso y ser muy cautos, aun a riesgo de dejar pasar una errata.

$\mathrm{Ni}$ que decir tiene que la edición sobre la que se realiza la concordancia puede contener variantes. De hecho, es ésta una característica que marca casi indeleblemente la edición original, frente a aquélla que es copia de otra. Voy a poner dos ejemplos.

En la línea 3 de la pág. 63 del tomo v Fog edita “revierte” allí donde Garagorri había editado "reviene”. Los editores de Fog no consignan ninguna variante en esta palabra, por lo que hemos de pensar que se trata de una errata de Garagorri. Subsanada la errata, “revenir" ya no se documenta en la obra de Ortega (hasta el momento): ésta era la única ocurrencia, según la edición de Garagorri.

\section{Editar en el siglo XXI}

La labor del editor no consiste, insistimos, en reproducir; para eso está el facsímil, que reproduce la edición que se hizo en su momento; y editar en 1914 no es editar en 2008. Los ejemplos podrían multiplicarse, pero sólo aduciremos uno más. De un total de 38 ocurrencias, los editores de Alianza (Garagorri) y Taurus (equipo de edición) escriben Dostoyewski (tomo IA, IIA, IT, IIIT, IVT), Dostoievski (tomo IIA, IIT), Dostoievsky (IIA), Dostoyewsky (IA, IIIA, VIA). Aunque la oscilación fuera atribuible al autor, es tarea del editor regularizar, en una publicación unitaria, la grafía oscilante ${ }^{11}$.

\section{El caso de la traducción}

La cosa se complica si (no es el caso de la obra de Ortega) la edición es a la vez la traducción de un texto de otra lengua ${ }^{12}$. Los ejemplos son muy numerosos, y la mayoría de ellos muestran esta inconsistencia en los criterios de edición.

El lexicógrafo está supeditado al texto editado que sigue en su trabajo. En ocasiones, y ante inconsistencias en los criterios de edición, se verá obligado a remitir de una forma a

oscilaciones. No he sido capaz de localizar el pasaje. Quizá sea una expresión creada a partir de Aristóteles, Política 1253a 3 o( a)/nqrwpoj fu/sei politiko $\backslash n \mathrm{zw} \mid=$ on

${ }^{10}$ Tomo ii, pág. 355 Fog; tomo ii, pág. 226 Garagorri.

${ }^{11}$ Haciendo una búsqueda en Google, Dostoievski se documenta 278.000 veces; Dostoievsky 96.500; Dostoyewski 1.730 y Dostoyewsky 574. Es tarea del editor regularizar la grafía; sobre todo, cuando se reúnen publicaciones tan distantes en el tiempo. No nos estamos refiriendo a lo problemático que resulta la transcripción del nombre del autor ruso, sino a la necesidad de que el editor opte por una forma: lo otro no es editar, sino unir en aluvión las diferentes ediciones precedentes.

${ }^{12}$ Así, por ejemplo, Týche (gr. Tu/xh) aparece editado en Garagorri “Tyche”. En la traducción al castellano de $L a$ esencia de la tragedia griega de A.-J. Festugière (Ariel, Barcelona 1986 [1969], trad. de Miguel Morey) leemos "Tyché” (pág. 26), suponemos que por culpa del original francés. Por otro lado, el libro contiene abundantes citas en griego, que aparecen excelentemente editadas en su alfabeto original. 
otra (la canónica). Es lo que hemos hecho en la Concordantia Ortegiana, dado que el mismo término griego aparece editado unas veces en el alfabeto original, otras transliterado siguiendo unos criterios, otras transliterado conforme a criterios distintos; en esos casos, y mediante el hiperenlace $^{13}$, he remitido a la forma que, según criterios científicos al uso, debiera haber sido editada. En el caso concreto de la obra orteguiana, poco o nada se ha avanzado en esta dirección con la nueva edición. A título de ejemplo, mencionaremos el caso de la letra $\mathrm{f}$ del griego: unas veces vemos editado el término en el alfabeto original (filoqeamo/nej), otras mediante el dígrafo “ph” (philotheamones) y otras, por último mediante la grafía "f” (con y sin tilde: "filotheamón” / "filotheamones"; también “filoceamones”) ${ }^{14}$.

\section{Nuestra Concordantia}

El hecho de que nuestra Concordantia se base en una edición exclusivamente (la de Paulino Garagorri en Alianza Editorial) comporta también algunos inconvenientes. El más importante, en nuestra opinión, es la eventual posibilidad de que el texto en cuestión presente variantes ${ }^{15}$. No obstante, el texto de Ortega está fijado con bastante fiabilidad, a juzgar por la prueba a que hemos sometido una de sus obras, El tema de nuestro tiempo ${ }^{16}$.

\section{Variantes textuales en EI tema de nuestro tiempo}

\footnotetext{
${ }^{13}$ La edición digital tiene estas ventajas.

${ }^{14}$ Las citas, en la edición de Garagorri, se pueden localizar con ayuda de la Concordantia Orteguiana citada en la nota 2. La última forma citada, “filoceamones” (auténtico barbarismo) ha sido mantenida en la edición Fog (tomo v, pág. 207). Como no hay en ella ni una sola nota sobre el contenido, nada sabemos de la opinión de los editores al respecto.

${ }^{15}$ Lógicamente, la base de datos obvia este inconveniente, al permitirnos incluir variantes textuales. Vamos a intentar soslayar este inconveniente también en una concordancia tradicional. Hemos explicado el procedimiento seguido en Fresnillo Núñez, Javier - Biosca i Bas, Antoni. "Propuesta para la elaboración de una concordancia lematizada: la 'Institutio oratoria’ de Quintiliano”, apud Cifuentes Honrubia, J.L. - Marimón Llorca, C. (coord.), Estudios de lingüística: el verbo. Universidad de Alicante, Alicante 2004, págs. 207-214.

${ }^{16}$ Para obtener los datos que ofrecemos a continuación he digitalizado las ediciones de Paulino Garagorri (tomo iii, 1983), Domingo Hernández (Tecnos, Madrid 2002) y Fog. (tomo iii, 2005). A continuación, he realizado el OCR de las tres ediciones y revisado manualmente el resultado (no he colacinado los textos). Por último, he procedido a comparar los textos - dos a dos- con el programa CorelWordPerfect. Todo ello ha formado parte de un curso de Doctorado sobre La edición de textos en la Universidad de Alicante (curso 2005-06). Quiero agradecer al alumno de dicho curso, D. Jordi Gandía, el haber puesto a mi disposición el trabajo realizado por él con estos materiales. He dejado fuera el "Prólogo para alemanes", que aún no ha sido publicado en la edición de Fog. (en efecto, al seguir los editores un criterio cronológico rígido, Fog. no publica la obra al completo). Reproduzco la nota de Domingo Hernández (pág. 199) al respecto: “Obra póstuma publicada por primera vez en la Editorial Taurus (Madrid), en 1958. Cuatro años después se incluyó ya en el volumen VIII de las Obras completas, y, posteriormente, en 1974, Paulino Garagorri realizó una nueva edición en El Arquero utilizando el manuscrito y agregando (además de algunas correcciones) los dos últimos párrafos.”
} 
Al proceder a la comparación, nos da la impresión de que tanto la edición de Tecnos ${ }^{17}$ (en adelante, $H$ ) como la de Taurus ${ }^{18}(F)$ han partido de un estadio textual semejante al que ofrece la edición de Garagorri $(G)$. Las variantes (relativamente pocas, aunque significativas ${ }^{19}$ ) permiten establecer relaciones entre los textos: a veces $\mathrm{G}$ y $\mathrm{H}$ coinciden frente a F; otras veces, G y F coinciden frente a $\mathrm{H}$; en otras ocasiones, $\mathrm{H}$ y $\mathrm{F}$ presentan un texto distinto de G. En este último caso es casi seguro que estamos ante erratas que se han deslizado en $\mathrm{G}$.

Pero el caso más llamativo, en nuestra opinión, se produce cuando G (Alianza) y H (Tecnos) presentan un texto coincidente, distinto del editado en $\mathrm{F}$, sin que los editores de Fog. consignen ningún tipo de variante. Es muy probable que tanto Paulino Garagorri como Domingo Hernández estén reproduciendo la misma versión del texto orteguiano, pero reconocemos que nosotros no hemos hecho la pesquisa correspondiente ${ }^{20}$.

\subsection{Coincidencias entre la edición de D. Hernández (H) y Fog. (F) frente a la edición de P. Garagorri (G)}

Veamos los datos, agrupados convenientemente.

\begin{tabular}{|l|l|}
\hline $559,5^{21}$ & D. G: don FH \\
\hline 567,1 & pensamiento G: pensamientos HF \\
\hline $567,-5$ & rigurosa G: rigorosa HF \\
\hline 569,15 & verdaderas G: verdades HF \\
\hline 569,21 & acero G: arco HF \\
\hline 571,7 & leve om. G \\
\hline 572,14 & el G: al HF \\
\hline 575,25 & fué G: fue FH \\
\hline 576,12 & elaborados G: elaborado HF \\
\hline $578,-7$ & Neftali G: Neftalí HF \\
\hline 585,11 & naturalismo G: naturalista HF \\
\hline $587,-6$ & objetivizado G: objetivado HF \\
\hline $590,-9$ & ser HF: om. G \\
\hline $594,-8$ & de vitalismo HF: del vitalismo G \\
\hline $594,-7$ & primitivo om. G \\
\hline
\end{tabular}

\footnotetext{
17 "Esta edición de El tema de nuestro tiempo sigue la tercera de 1934 (Revista de Occidente, Madrid), anotando a pie de página las variantes tanto respecto a la primera de 1923 (Calpe, Madrid), como a la publicación en la prensa de los distintos capítulos. También se han manejado las distintas ediciones en las Obras completas de Ortega. Si las diferencias entre las dos ediciones del libro se han anotado en detalle, respecto a los periódicos únicamente se señalan las diferencias más significativas.”, pág. 33.

${ }^{18}$ Sus editores han tomado como base los mismos textos que D. Hernández, a tenor de lo que señalan en la página 960.

${ }^{19}$ Hemos registrado 123 discrepancias, de un total de 37.637 palabras en la edición de Fog., lo que supone el $0,33 \%$.

${ }^{20}$ Se supone que la edición de Fog., en Taurus, debería habernos ayudado en este sentido.

${ }^{21}$ La referencia la hacemos por la edición de Fog. El primer número remite a la página, el segundo a la línea, teniendo en cuenta que contamos todas las líneas físicas con inclusión de títulos y numeración de apartados.
} 


\begin{tabular}{|l|l|}
\hline $594,-5$ & nulo G: nudo HF \\
\hline 596,15 & rigurosa G: rigorosa HF \\
\hline 598,34 & cristianismo G LN (1-V-23), TNT (23): cristiano \\
\hline 599,2 & obliga G: obligaba HF \\
\hline 599,7 & ha G: han HF \\
\hline 599,12 & aptitud G: actitud HF \\
\hline $600,-13$ & Esta G: Esa HF \\
\hline 603,6 & rigurosamente ${ }^{22}$ G: rigorosamente HF \\
\hline $604,-4$ & el [sc. él] fue G: que fue HF \\
\hline 609,8 & vitalidad G: finalidad HF \\
\hline 611,8 & desvanecer G: desvanece HF \\
\hline $611,-6$ & insuficientes G: suficientes HF \\
\hline 612,26 & acontecía G: acontecería HF \\
\hline 613,39 & indomable G LN (12-VIII-23), O II (32), O (36), O \\
\hline 614,19 & II (43): indominable HF \\
\hline $622,-12$ & transmitidos G: transmitido HF \\
\hline $623,-10$ & constituído G: constituido HF \\
& constituído G: constituido HF \\
\hline 628,15 & obligan G: obligaban HF \\
\hline 628,23 & Hocsent G: Hocsem HF \\
\hline 632,4 & hace G: no hace HF \\
\hline $633,-10$ & tiempo G: tiempos HF \\
\hline $634,-9$ & lo G: la HF \\
\hline $634,-2$ & están G: serán HF \\
\hline $635,-4$ & algunos G: algunas HF \\
\hline 637,19 & lema G: el lema HF \\
\hline 639,5 & tradicionalista G: racionalista HF \\
\hline 640,20 & general G: universal HF \\
\hline & \\
\hline
\end{tabular}

Podemos afirmar que, en la mayoría de los casos, estamos ante erratas subsanadas. Claros ejemplos son las lecturas de 569,21 acero G: arco HF; 578,-7 Neftali G: Neftalí HF; 585,11 naturalismo G: naturalista HF; 594,-5 nulo G: nudo HF; 599,12 aptitud G: actitud HF; 611,-6 insuficientes G: suficientes HF; 628,23 Hocsent G: Hocsem HF. En unas pocas ocasiones las variaciones son mínimas, y obedecen a la modificación de las normas de acentuación: 575,25 fué G: fue FH; 623,-10 constituído G: constituido HF. Son meras cuestiones gráficas, sin importancia para el establecimiento del texto. Hay más variantes de

\footnotetext{
${ }^{22}$ Ricardo Senabre afirma taxativamente que las formas en "rigu-” son erratas, que Ortega escribió siempre "rigo-”, en su reseña de los tomos i y ii de Fog.: http:/www.elcultural.es/html/20041125/letras/LETRAS10754.asp (13/09/2006). [consulta: 16/09/2006; ya no está accesible por Internet] En la edición de Garagorri, rigurosa se documenta en 14 ocasiones (frente a rigorosa, 132), rigurosamente en 13 (rigorosamente, 142), rigurosas en 2 (rigorosas, 32), riguroso en 14 (rigoroso,135), rigurosos en 4 (frente a rigorosos, 23). Los superlativos son editados siempre bajo la forma "rigo-": rigorosísima en 4 ocasiones, rigorosísimas en 1 y rigorosísimos en 1 ocasión. Vid. Concordantia Ortegiana s.v.
} 
este tipo que no hemos recogido, por carecer de importancia (son cuestiones ortográficas, no significativas por tanto).

Pero hay una serie de lecturas que no parecen deberse a meras erratas, sino a variantes textuales. No hemos indagado en esta dirección. Eso sí, hemos recogido los datos que sobre variantes aporta la edición de Fog.

\subsection{Coincidencias entre la edición de P. Garagorri (G) y Fog. (F) frente a la edición de D. Hernández (H)}

Veamos a continuación las coincidencias.

\begin{tabular}{|l|c|}
\hline 562,14 & terra incognita GF: “terra incognita” H \\
\hline $570,-8$ & flúido GF: fluido H \\
\hline 573,19 & Pio GF: Pío H \\
\hline 573,20 & Pio GF: Pío H \\
\hline 573,26 & «la razón» GF: la «razón» H \\
\hline $574,-6$ & mismo GF: misma H \\
\hline $574,-2$ & raison GF: «raison» H \\
\hline 575,2 & pura» GF: pura», H \\
\hline 575,17 & sólidamente GF: tan sólidamente H ES (24-I-23), \\
& TNT (23, 34), O II (32), O (36), O II (43), OC(47) \\
\hline 576,12 & «lógica maravillosa» GF: «lógica» maravillosa H \\
\hline 581,23 & ejercitan GF: ejercen H \\
\hline 582,26 & abstraigan GF: abstraiga H \\
\hline 585,24 & esas GF: om. H ES (9-II-23), TNT (23, 34), O II \\
& (32), O (36), O II (43) \\
\hline 586,30 & Se creía GF: Se creía que se creía H ES (9-II-23), \\
& TNT (23, 34), O II (32), O (36), O II (43) \\
\hline $587,-5$ & contra-puesto GF: contrapuesto H \\
\hline 590,17 & de GF: en H \\
\hline $593,-3$ & con GF: om. H \\
\hline 596,35 & en él expresa GF: expresa en él H LN (1-V-23), \\
& TNT (23, 34), 0 II (32), O (36), O II (43) \\
\hline 598,15 & tributamos GF: tributemos H \\
\hline 599,27 & positivistas GF: positivas H TNT (23, 34), O II \\
& (32), O (36), O II (43): postreras LN (1-V-23) \\
\hline 600,27 & ilusoria GF: ilusionaria H LN (1-V-23), TNT (23, \\
& 34), O II (32), O (36), O II (43), OC (47) \\
\hline 602,10 & sobre-vivirse, GF: sobrevivirse H \\
\hline $602,-1$ & subordina GF: subordinan H \\
\hline 603,4 & zoé GF: zoe H \\
\hline 603,4 & bíos GF: bios H \\
\hline 604,4 & los GF: sus H \\
\hline $606,-1$ & del GF: de un H \\
\hline 608,17 & semi-religioso GF: semirreligioso H \\
\hline & \\
\hline & \\
\hline & \\
\hline & \\
\hline &
\end{tabular}




\begin{tabular}{|c|c|}
\hline 608,20 & toma GF: toman $\mathrm{H}$ \\
\hline 609,10 & esperanzas GF: esperanza $\mathrm{H}$ \\
\hline 612,14 & ser GF: vez H \\
\hline 616,20 & atribuimos (atribuímos G) GF: atribuíamos H \\
\hline 620,15 & acontecimientos GF: conocimientos H \\
\hline 621,4 & angustia de GF: angustia en $\mathrm{H}$ \\
\hline 621,4 & tiranía de GF: tiranía en $\mathrm{H}$ \\
\hline 621,10 & Danton GF: Dantón H \\
\hline 622,14 & kanguros GF: canguros $\mathrm{H}$ \\
\hline 627,21 & la vida GF: vida $H$ \\
\hline $629,-11$ & quien $\mathrm{GF}$ : que $\mathrm{H}$ \\
\hline $629,-8$ & utopismos GF: utopismo $\mathrm{H}$ \\
\hline 630,23 & su presión GH: presión H \\
\hline 631,11 & ha GF: han $\mathrm{H}$ \\
\hline 631,13 & extenderá GF: extenderán $\mathrm{H}$ \\
\hline 631,13 & del Norte GF: el Norte H \\
\hline 631,29 & $\begin{array}{l}\text { estado GF: estadio H ES (17-VII-23), TNT }(23,34) \text {, } \\
\text { O II (32), O (36), O II (43) }\end{array}$ \\
\hline $631,-10$ & el cuerpo GF: cuerpo H \\
\hline 633,14 & Chrémata GF: Chremata H \\
\hline 633,14 & chrémata GF: chremata H \\
\hline 633,16 & hallemos GF: hallamos $\mathrm{H}$ \\
\hline 634,13 & mythos GF: mithos H \\
\hline $634,-11$ & la GF: om. $H$ \\
\hline 643,16 & creen en GF: creen H \\
\hline 644,7 & tempo-espacial GF: tempoespacial H \\
\hline 646,6 & $\begin{array}{l}\text { imaginación GF: imagen H LN (9-IX-23), TNT } \\
\text { (23), O II (32), O (36), O II (43) }\end{array}$ \\
\hline 646,13 & al GF: a mi H \\
\hline 646,20 & una sola GF: un sola $\mathrm{H}$ \\
\hline 648,18 & su puesto GF: supuesto $\mathrm{H}$ \\
\hline 649,1 & realidad, y, GF: realidad y, H \\
\hline 649,20 & estos GF: esos H \\
\hline 651,3 & dictadora GF: dictadura $\mathrm{H}$ \\
\hline $652,-15$ & metron GH: métron F \\
\hline
\end{tabular}

Los casos en que nos encontramos ante meras erratas son muy pocos: 573,19 Pio GF: Pío H (el texto está en italiano); 643,16 creen en GF: creen H; 648,18; su puesto GF: supuesto $\mathrm{H}$; 646,20 una sola GF: un sola $\mathrm{H}$; 651,3 dictadora GF: dictadura $\mathrm{H}$. En algún caso mejora la lectura de la edición de Fog.: 652,-15 metron GH: métron F. Pero en muchas más ocasiones tenemos la impresión de que D. Hernández está siguiendo una edición distinta de la seguida por Garagorri y Fog., sin que los editores que lo hacen (Hernández y, sobre todo, Fog.) recojan variantes de ningún tipo. 


\subsection{Recogemos, por último, pasajes en que las ediciones de Garagorri (G) y Hernández (H) presentan un texto coincidente frente al de Fog. (F)}

Veamos a continuación los pasajes.

\begin{tabular}{|l|l|}
\hline 562,14 & la terra GH: terra F \\
\hline $564,-11$ & etc. GH: etcétera F \\
\hline $569,-11$ & época F: etapa GH \\
\hline $569,-6$ & proclamado GH: reclamado F \\
\hline 575,7 & etc. GH: etcétera F \\
\hline 585,22 & hallan GH: hallen F \\
\hline $585,-12$ & adhiriendo GH: adherido F \\
\hline $599,-6$ & etc. GH: etcétera F \\
\hline 602,6 & interiores, y GH: interiores y, F \\
\hline 603,14 & la GH: lo F \\
\hline 604,4 & etc. GH: etcétera F \\
\hline 607,6 & suficiente GH: suficientes F \\
\hline 609,22 & simpar GH: sin par F \\
\hline 626,11 & constitución GH: construcción F \\
\hline 628,18 & privilegios GH: privilegiados F \\
\hline 633,22 & donde GH: adonde F \\
\hline $634,-13$ & Möllendorf GH: Moellendorff F \\
\hline 635,23 & numerar GH: enumerar F \\
\hline 637,1 & J.C. GH: Jesucristo F \\
\hline 637,13 & Lafayette GH: La Fayette F \\
\hline 651,9 & etc. GH: etcétera F \\
\hline & \\
\hline
\end{tabular}

En dos ocasiones (562,14 la terra GH: terra F; 602,6 interiores, y GH: interiores y, F) creo que se trata de una errata en la edición de Fog. En otros casos (575,7 etc. GH: etcétera F; 637,1 J.C. GH: Jesucristo F) los editores de Fog. parecen estar resolviendo las abreviaturas. Creemos que hay que destacar el esfuerzo de Fog. por limpiar el texto de erratas (585,-12 adhiriendo GH: adherido F; 609,22 $\operatorname{simpar}^{23} \mathrm{GH}$ : sin par F). En el caso de 637,13 Lafayette GH: La Fayette F, son aceptables ambas formas del nombre ${ }^{24}$. Ante la discrepancia de Fog., sin que registre variantes, nos quedamos sin saber cuál fue la grafía empleada por Ortega. No sucede lo mismo en el caso de 634,-13 Möllendorf GH: Moellendorff F. La oscilación “ö/oe” es una cuestión menor, meramente ortográfica, pero la forma correcta del nombre del célebre filólogo alemán es "Moellendorff”, con dos efes. Es curioso, en este sentido, que Domingo Hernández, a pesar de haber anotado debidamente el

\footnotetext{
${ }^{23}$ Esta forma la edita Garagorri hasta en cinco ocasiones. Ni Domingo Hernández ni nosotros en nuestro trabajo de lematización llegamos a ver la errata.

${ }^{24}$ Recuérdese, en este sentido, el nombre de las famosas “Galeries Lafayette”, en Francia.
} 
personaje citado por Ortega, escriba “Möllendorf”. ¿Será esta la grafía empleada por Ortega? ${ }^{25}$.

Pero siguen restando un cierto número de pasajes en que da la impresión de que los editores hayan optado por variantes distintas, sin que haya quedado constancia de ello en las respectivas ediciones.

6.4. Pasajes en que las tres ediciones $(G, H, F)$ presentan un texto no coincidente entre sí.

Veamos a continuación los pasajes.

\begin{tabular}{|l|l|}
\hline 608,29 & anularlo F: anularle G: anularla H \\
\hline 633,14 & anér G: áner H: aner F \\
\hline 651,8 & Lobacchewski G: Lobachevski F: Lobatchewski H. \\
\hline
\end{tabular}

En los tres últimos casos, finalmente, los editores de Fog. editan un texto distinto del editado por H (Domingo Hernández) y G (Garagorri), sin que los editores dejen constancia de que existen divergencias entre los textos orteguianos. En 608,29 la discrepancia a tres bandas es aparente; en efecto, si contamos con el leísmo que mencionan los editores de Fog. las lecturas de $\mathrm{G}$ y de $\mathrm{F}$ son coincidentes. La lectura de $\mathrm{H}$ es o bien una errata, o bien una variante (sin registrar). El caso de 633,14 es un buen ejemplo del comportamiento vacilante de los editores frente a la transcripción de términos griegos, oscilación que sale a la luz cuando el investigador procede a lematizar el texto.

En 651,8 creo que la lectura correcta es la de H ("Lobatchewski”). Las lecturas de G y de $\mathrm{H}$ serían, a ese tenor, erratas. Aunque bien pudiera ser que la lectura "original” fuera la de G ("Lobacchewski”), ya que a partir de ella es posible explicar - por error gráfico- tanto la lectura de H (Hernández habría interpretado correctamente que "cc” es una errata, por “tc”) como la de Fog., que sería una lectio facilior, con la simplificación del grupo consonántico.

\section{Conclusiones}

A la vista de los hechos anteriores, pensamos que cabe la posibilidad de que los editores no hayan recogido la totalidad de las variantes que presenta el texto de Ortega y Gasset. En efecto, las coincidencias entre la primera de las ediciones de El tema de nuestro tiempo que hemos traído a colación, obra de Paulino Garagorri, y la última de ellas, obra del equipo de investigación de la Fundación Ortega y Gasset, sugieren que no estamos ante meras erratas (como nos parece que es el caso de las variantes recogidas en apartado 6.1) sino ante

\footnotetext{
${ }^{25}$ Estaríamos, en este caso, ante una falta de autor. El nombre lo edita Garagorri en 8 ocasiones, siempre con una sola efe. Para saberlo, habríamos de disponer de un autógrafo de Ortega, puesto que siempre cabe la posibilidad de que se trate de una errata de imprenta, si bien en este último caso lo habitual es que encontremos oscilaciones entre ambas grafías.
} 
verdaderas variantes textuales. Desgraciadamente, la información de los editores al respecto es más parca de lo que requiere la acribia filológica.

Tal es la problemática con la que ha de enfrentarse el lexicógrafo a la hora de acometer la elaboración de una concordancia lematizada. Cuanto más "verosímil" sea el texto editado, tanto mayores son las posibilidades de que las erratas pasen desapercibidas ${ }^{26}$. Los barbarismos son más fáciles de detectar: si en un texto nos encontramos con Pío inserto en una cita en italiano, es presumible que estemos ante una errata. El objetivo del investigador que está lematizando no es editar el texto, pero sí tiene obligación de dejar constancia de aquellos términos que puedan constituir un texto erróneo. Aun a riesgo de equivocarse. Eso sí: con sumo cuidado. Hay que pensar que quien más y mejor conoce el texto editado es el propio editor.

\section{Referencias bibliográficas}

Fresnillo Núñez, Javier - Pérez Herranz, Fernando M. (col.). Concordantia Ortegiana. Concordantia in José Ortega y Gasset opera omnia. Universidad, Alicante 2004.

Fresnillo Núñez, Javier - Pérez Herranz, Fernando Miguel, "Presentación de la concordancia de las Obras completas de José Ortega y Gasset” Revista de estudios orteguianos 3 (2001) 129-146.

Fresnillo Núñez, Javier - Pérez Herranz, Fernando Miguel, "Ortega y los siervos de la gleba cultural” El Basilisco, 2. ${ }^{a}$ época, 32 (2002) 3-10.

Fresnillo Núñez, Javier - Biosca i Bas, Antoni. "Propuesta para la elaboración de una concordancia lematizada: la 'Institutio oratoria' de Quintiliano”, apud Cifuentes Honrubia, J.L. - Marimón Llorca, C. (coord.), Estudios de lingüística: el verbo. Universidad de Alicante, Alicante 2004. Págs. 207-214.

Ortega y Gasset, José. El tema de nuestro tiempo. Ed. Domingo Hernández Sánchez. Tecnos, Madrid 2002.

Ortega y Gasset, José. Obras completas. Tomo i (1902-1915). Ed. Fog. (Carmen Asenjo Pinilla, Ignacio Blanco Alfonso, José Ramón Carriazo Ruiz, María Isabel Ferreiro Lavedán, Iñaki Gabaráin Gaztelumendi, Azucena López Cobo, Juan Padilla Moreno, Javier Zamora Bonilla), Taurus - Revista de Occidente, Madrid 2004.

Ortega y Gasset, José. Obras completas. Tomo ii (1916). Ed. Fog. (Carmen Asenjo Pinilla, Ignacio Blanco Alfonso, José Ramón Carriazo Ruiz, María Isabel Ferreiro Lavedán, Iñaki Gabaráin Gaztelumendi, Azucena López Cobo, Juan Padilla Moreno, Javier Zamora Bonilla), Taurus Revista de Occidente, Madrid 2004.

Ortega y Gasset, José. Obras completas. Tomo iii (1917-1925). Ed. Fog. (Carmen Asenjo Pinilla, Ignacio Blanco Alfonso, José Ramón Carriazo Ruiz, María Isabel Ferreiro Lavedán, Iñaki Gabaráin Gaztelumendi, Azucena López Cobo, Juan Padilla Moreno, Javier Zamora Bonilla), Taurus - Revista de Occidente, Madrid 2005.

Ortega y Gasset, José. Obras completas. Tomo v (1932-1940). Ed. Fog. (Carmen Asenjo Pinilla, Ignacio Blanco Alfonso, José Ramón Carriazo Ruiz, María Isabel Ferreiro Lavedán, Iñaki

\footnotetext{
${ }^{26}$ Es lo que nos ha sucedido a nosotros en ocasiones; por ejemplo, con “adhiriendo”, que al ser detectada en la comparación entre las ediciones nos parece una errata evidente, pero que no detectamos a la hora de preparar la base de datos del texto orteguiano, ni tampoco en el proceso de lematización.
} 
Gabaráin Gaztelumendi, Azucena López Cobo, Juan Padilla Moreno, Javier Zamora Bonilla), Taurus - Revista de Occidente 2006.

Ortega y Gasset, José. Obras completas. Tomo vii (obra póstuma, 1902-1925). Ed. Fog. (Carmen Asenjo Pinilla, Cristina Blas Nistal, José Ramón Carriazo Ruiz, María Isabel Ferreiro Lavedán, Iñaki Gabaráin Gaztelumendi, Felipe González Alcázar, Azucena López Cobo, Javier Zamora Bonilla.

Ortega y Gasset, José. Obras completas. 12 vols. Ed. Paulino Garagorri, Alianza editorial, Madrid 1983. 